\title{
BMJ Open Protocol for a randomised, double- blind, placebo-controlled trial to explore the effect of tramadol in the prevention of postpartum depression (ETPPD)
}

\author{
Guangyou Duan, ${ }^{1}$ Zhuoxi Wu, ${ }^{1}$ Peng Zhao, ${ }^{1}$ Jing Peng, ${ }^{1}$ Zhengqiong Chen, ${ }^{2}$ \\ Qingling Zhang, ${ }^{3}$ Rufu Xu, ${ }^{4}$ Hong Li ${ }^{1}$
}

To cite: Duan G, Wu Z, Zhao P, et al. Protocol for a randomised, double-blind, placebo-controlled trial to explore the effect of tramadol in the prevention of postpartum depression (ETPPD). BMJ Open 2018;8:e022538. doi:10.1136/ bmjopen-2018-022538

- Prepublication history and additional material for this paper are available online. To view these files, please visit the journal online (http://dx.doi. org/10.1136/bmjopen-2018022538).

Received 24 February 2018 Revised 13 June 2018 Accepted 14 September 2018

Check for updates

(c) Author(s) (or their employer(s)) 2018. Re-use permitted under CC BY-NC. No commercial re-use. See rights and permissions. Published by BMJ.

${ }^{1}$ Department of Anesthesiology, Xinqiao Hospital, Third Military Medical University, Chongqing, China

${ }^{2}$ Department of Obstetrics, Xinqiao Hospital, Third Military Medical University, Chongqing, China

${ }^{3}$ Department of Psychology, Xinqiao Hospital, Third Military Medical University, Chongqing, China

${ }^{4}$ Department of Health Statistics, Third Military Medical University, Chongqing, China

Correspondence to

Dr Hong Li; lh78553@163.com

\section{ABSTRACT}

Introduction Postpartum depression is one of the most commonly experienced psychological disorders for women patients undergoing caesarean section, which accounts for about one-third of puerpera worldwide. Tramadol, a commonly used analgesic with an inhibitory effect on the reuptake of norepinephrine and serotonin, is an effective and well-tolerated agent for analgesia after caesarean section. Based on the role of changes in the postpartum levels of serotonin and norepinephrine in postpartum depression, we speculated that postoperative intravenous analgesia using tramadol may decrease the incidence of postpartum depression for caesarean patients. Therefore, this trial aimed to explore the effect of tramadol in the prevention of postpartum depression.

Methods and analysis A randomised double-blind placebo-controlled trial will be performed and 1230 singleton parturients will be randomised to receive patient-controlled intravenous analgesia with tramadol or hydromorphone, or patient-controlled epidural analgesia with ropivacaine. The primary outcome of this trial will be the incidence of postpartum depression at 4 weeks after the caesarean section, together with the collection of the relevant data during hospitalisation and at 3 months after the caesarean section. Subgroup data according to the preoperative depression score will be analysed. Demographic characteristics, postoperative analgesic effects and postoperative recovery score will also be summarised and presented.

Ethics and dissemination The current trial protocol was approved by the Institutional Ethics Committee of Xinqiao Hospital (ID: 2017-026), Third Military Medical University, Chongqing, China. The results of this trial will be disseminated at scientific meetings and in a peer-reviewed journal.

Trial registration number NCT03309163; Pre-results.

\section{INTRODUCTION}

Postpartum depression is one of the most commonly experienced psychological illnesses for puerperas 'within peripartum onset if onset of mood symptoms occurs during pregnancy or within 4 weeks following delivery' (American Psychiatric Association's
Strengths and limitations of this study

- The current trial is the first to explore the possible effect of patient-controlled intravenous analgesia (PCIA) using tramadol for prevention of postpartum depression for patients who have received elective caesarean section.

- A total of 1230 patients who are scheduled to receive elective caesarean section will be included in the current trial, and the incidence of postpartum depression will be evaluated at 4 weeks and 3 months after the surgery.

- Stratified block randomisation will be performed according to the preoperative psychological test, and subgroup analysis will be performed in the final data analysis.

- Two commonly used analgesic strategies including PCIA using hydromorphone and patient-controlled epidural analgesia (PCEA) using ropivacaine will be used as control groups.

- Because of the obvious difference between PCIA and PCEA treatments, complete masking of the participant or the investigator during the hospitalisation follow-up is not feasible. However, investigators who are responsible for postoperative long-term follow-up after discharge and the data analyser are unaware of the group allocation.

Diagnostic and Statistical Manual of Mental Disorders, Fifth Edition, DSM-5) or 'within 6 weeks following delivery' (International Classification of Diseases, 10th Revision). ${ }^{12}$ The estimated prevalence of postpartum depression in higher income countries is about $10 \%$ and even higher in lower income and middle income countries ${ }^{2}{ }^{3}$; it has been reported that in China it reaches 13.7\%$15.7 \% .^{45}$ Symptoms of postpartum depression often include sleep disturbance, anxiety, irritability and a feeling of being neglected, as well as an obsessional preoccupation with the baby's health and feeding. Suicidal ideation and causing harm to the baby have also been 
reported. ${ }^{6}$ Some studies have even found that these symptoms may continue for up to 2 years postpartum without prevention or treatment, and approximately $40 \%$ of affected women will have a relapse during their subsequent pregnancies. ${ }^{8}$ However, at present, studies regarding the prevention of postpartum depression for women patients undergoing caesarean section remain lacking. ${ }^{9}$

Caesarean section is one of the most commonly used strategies for maternal delivery all over the world. A WHO survey has shown that the global caesarean section rate was $27.3 \%$ in $2008 .{ }^{10}$ The latest data show that the caesarean section rate in China reached $34.9 \%$ in 2014, ${ }^{11}$ and in the USA it reached $32.2 \%$ in $2014 .{ }^{12}$ These data indicate that there are tens of millions of maternal deliveries by caesarean section every year in the world. However, previous studies have demonstrated that the risk of postpartum depression after caesarean section is significantly higher than that of vaginal delivery. ${ }^{13-15}$ This might be associated with the long-standing belief that a 'normal birth' is a 'vaginal birth', ${ }^{1617}$ and another possible reason is that caesarean birth is often more painful and stressful. ${ }^{18}$ Therefore, postpartum depression for women undergoing caesarean section may need more attention and treatment.

Although the specific mechanism of postpartum depression is still unclear, the current data provide evidence that the underlying mechanism is likely to be associated with changes in related hormones, which is due to the rapid decline during postpartum reproductive period. ${ }^{19-22}$ At present, the treatment of postpartum depression mainly includes psychological and supportive therapy that is suitable for early intervention, and the effect of this treatment needs to be improved. ${ }^{123}$ Beyond these behavioural interventions, commonly used drugs for postpartum depression are mainly selective serotonin reuptake inhibitors (SSRI) and serotonin/norepinephrine reuptake inhibitors (SNRIs) but there is still limited knowledge of therapeutic effects and drug safety during breast feeding. ${ }^{24}{ }^{25}$ Based on the above information, the present drug treatment strategy of postpartum depression is often confined to an intervention after the onset of symptoms, but at present relatively less potential strategy has been explored or reported to prevent postpartum depression.

In addition to increased risk of postpartum depression, another issue experienced by patients undergoing caesarean section is postoperative pain induced by the surgery. ${ }^{26}$ Moreover, postoperative pain is the one of identified risk factors for depression. ${ }^{28}$ Patient-controlled intravenous analgesia (PCIA) with different analgesics is one of the most commonly used methods to treat postoperative pain by the clinical route. ${ }^{29-31}$ Among the commonly used analgesics, tramadol is an effective and well-tolerated agent for analgesia after caesarean section and it has been demonstrated as having the advantages of fewer adverse effects than powerful opioids. ${ }^{32}$ Previous studies demonstrated that tramadol administration via PCIA or intermittent intravenous infusion provides effective postoperative analgesia for patients who received a caesarean section. ${ }^{33-36}$ Furthermore, a recent study found that for primiparas following caesarean section, PCIA with tramadol might be an optimised strategy due to less pain at mobilisation, higher quality of recovery (Qor) and earlier lactation than sufentanil. ${ }^{37}$

Tramadol, a non-opioid central analgesic, has an inhibitory effect on the reuptake of norepinephrine and serotonin, indicating that it has the potential to reduce the risk of suffering from depression for puerperas. Furthermore, previous studies have demonstrated that in patients with postpartum depression the significant change of serotonin and norepinephrine levels presented at postpartum 48 hours, which matched well with the time period for postoperative analgesia. ${ }^{38}$ Thus, previous evidence could support the idea that postoperative 48 hours PCIA with tramadol has the potential to effect remission from postpartum anxiety and depression. In addition, as we know sertraline (SSRIs) is often recommended as firstline therapy in new-onset postpartum depression. ${ }^{40}$ And SNRIs are commonly used either when SSRIs are ineffective or when a woman has previously had a positive response to these agents. ${ }^{1}$ Thus, these traditional drug therapies are only recommended when symptoms are severe and require rapid treatment or when symptoms of postpartum depression do not resolve with psychological treatment alone. ${ }^{41}$ Different from these drug therapies, tramadol can serve as analgesic to treat postoperative pain after the caesarean section, and meanwhile it has an inhibitory effect both on the reuptake of norepinephrine and serotonin. Thus, compared with traditional antidepressant drug, postoperative analgesia using tramadol may be the one of the ideal strategies for caesarean patients in the presence of analgesic and antidepressant demand. Also, tramadol may beneficial for women who undergo vaginal delivery, which can be further explored in the future.

Furthermore, in our previous study (under submission), which was designed to compare the analgesia effects of PCIA using tramadol and hydromorphone for caesarean section during the 48 hours after the surgery. In tramadol group, tramadol $4 \mathrm{mg} / \mathrm{kg}$ and flurbiprofen $4 \mathrm{mg} / \mathrm{kg}$ mixed with $0.9 \%$ saline to a volume of $150 \mathrm{~mL}$ were placed in a PCIA pump, while hydromorphone $0.04 \mathrm{mg} /$ $\mathrm{kg}$ and flurbiprofen $4 \mathrm{mg} / \mathrm{kg}$ were placed in patients of group $\mathrm{H}$. The PCIA pump $(150 \mathrm{~mL})$ was programmed to have a background infusion at $2.0 \mathrm{~mL} /$ hours, a PCIA dose of $1 \mathrm{~mL}$ and a lockout period of $15 \mathrm{~min}$. We unexpectedly found that tramadol can significantly decrease the anxiety and depression scores of Hospital Anxiety and Depression Scale (HADS) at 1 week after the surgery. These results also indicated that tramadol may have the potential to decrease the risk of postpartum depression.

Based on the above information, we hypothesise that PCIA with tramadol may be an optional analgesic strategy for the prevention and treatment of postpartum depression for patients undergoing caesarean section. Therefore, the current study aimed to apply a randomised 


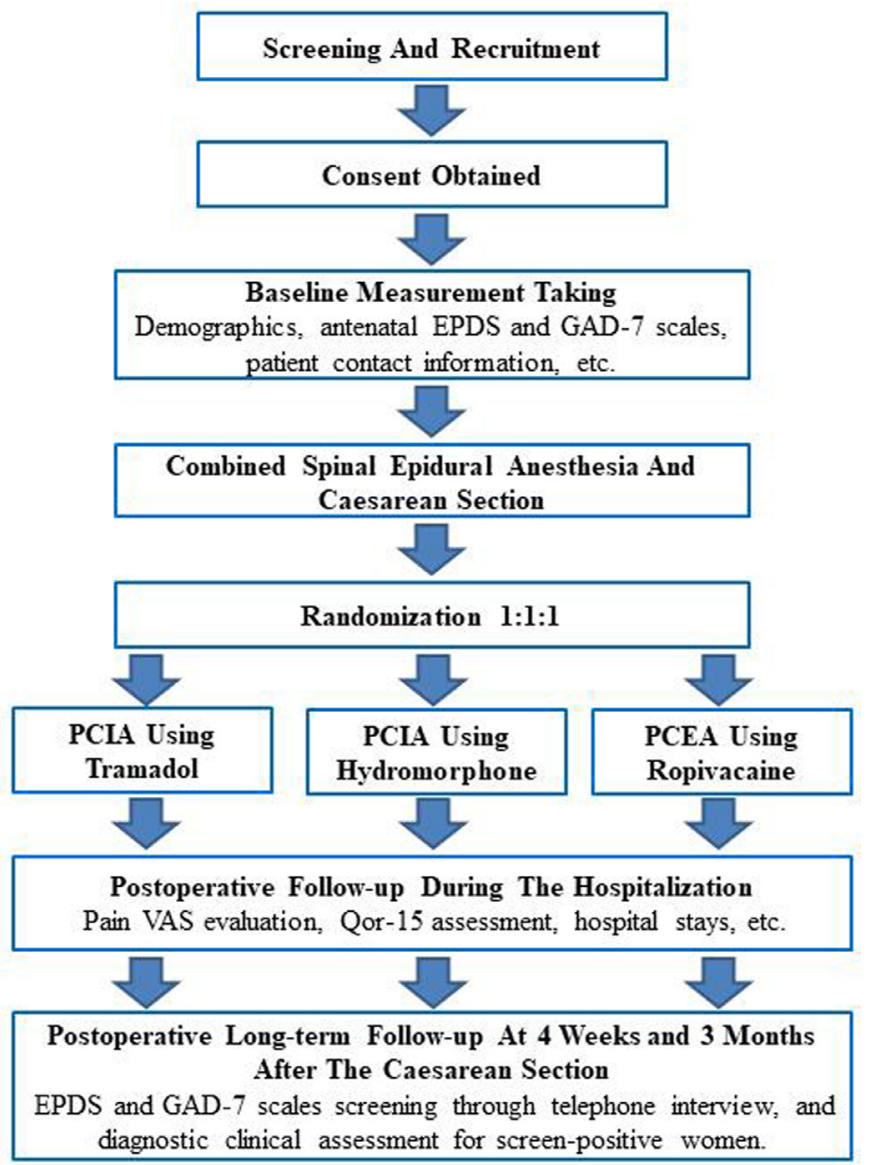

Figure 1 Study flow diagram. EPDS, Edinburgh Postnatal Depression Scale; GAD-7, Generalised Anxiety Disorder 7-item; PCIA, patient-controlled intravenous analgesia; PCEA, patient-controlled epidural analgesia; Qor-15, quality of recovery 15 items; VAS, Visual Analogue Scale.

controlled double-blind trial to include women patients who were undergoing caesarean section. Using psychological evaluation, the study also aimed to explore the possible effect of PCIA with tramadol for the prevention or treatment of postpartum depression in caesarean section, compared with PCIA with hydromorphone and patient-controlled epidural analgesia (PCEA).

\section{METHODS AND ANALYSIS \\ Trial design}

The study is designed as a prospective randomised controlled study and will be carried out according to the Consolidated Standards of Reporting Trials recommendations. ${ }^{42}$ The overview of the study protocol is shown in figure 1 , and the study setting is Xinqiao Hospital, Third Military Medical University, Chongqing, China. The recruitment phase of this study will last for about 18 months, beginning October 2017. Patients will be first screened in the obstetric ward and recruited according to the inclusion and exclusion criteria when admitted to hospital before the surgery. Before surgery, patient demographic characteristics will be recorded and the study procedure will be explained to all patients. The Edinburgh Postnatal Depression Scale (EPDS) and Generalised Anxiety Disorder 7-item (GAD-7) will be evaluated by investigator PZ. Then patients will be allocated to different groups according to the different postoperative analgesic strategies investigated, either PCIA using tramadol, PCIA using hydromorphone or PCEA using ropivacaine. The allocation will be determined by stratified block randomisation (performed by GD).

Electrocardiography, mean arterial pressure and pulse oxygen saturation will be monitored after patients enter the operating room. Standardised combined spinal-epidural anaesthesia (CSEA) will be performed by an experienced anaesthetist. Lumbar anaesthesia combined needle will be administered in the L3-L4 interspace, with an initial spinal dose of $1 \%$ ropivacaine $3 \mathrm{~mL}$. Then, epidural catheters will be placed into the epidural space to ensure that a sensory block up to T6 level with additional epidural infusion if needed. Surgery for included patients will be conducted by an experienced surgical team using standardised techniques. After the surgery, patients will be monitored for 6 hours in the postanaesthesia care unit before returning to the obstetric ward. The PCIA or PCEA treatment will be started immediately after surgery using an electronically controlled infusion pump.

Postoperative follow-up during the hospitalisation period was performed by JP and a long-term structured interview will be performed via telephone by ZW. We will get access to the 'WeChat' (the most commonly used social networking software in China) account of all the included women, and send the self-report questionnaires (EPDS and GAD-7) as well as text messages to schedule the appointments of telephone interviews by WeChat. Efforts will be made to reach all the included patients at 4 weeks and 3 months post partum. If a patient is not contacted for 1 week, she will be considered as lost to follow-up and no further contact will be attempted. The telephone interviewer has been trained to make an interview according to the established contents and order: (1) During the past month, have you often been bothered by feeling down, depressed or hopeless? And have you often been bothered by little interest or pleasure in doing things? (2) Please complete the self-report EPDS and GAD-7 questionnaires as you did during the hospitalisation before caesarean section. (3) Patient's pain status and any complications of the mothers and their infants after the surgery.

Patients deemed to be at risk of depression (screen positive according to EPDS) will be invited to undergo a diagnostic clinical assessment by telephone using the Structured Clinical Interview for DSM-5 (SCID-5) by a professional psychologist (QZ), who is unaware of the group allocation. Women who met DSM-5 criteria for major depressive disorder will be recorded as a positive case. Appropriate options for the treatment of postpartum depression will be decided by $\mathrm{QZ}$ and vary according to the severity of the patient's symptoms and her functional 
status, including her ability to care for, and relate to, the newborn. ${ }^{43}$ Finally, the data will be collected and imputed through Epidata software V.3.1 (Odense, Denmark) and the data analysis will be performed by a statistician from Department of Health Statistics of Third Military Medical University, who is also unaware of the details of the trial.

\section{Patients}

Chinese patients, aged 20-40 years, who are scheduled to receive elective caesarean section will be recruited into the current study. Inclusion and exclusion criteria are listed below.

\section{Inclusion criteria}

- Aged 20-40 years.

- American Society of Anesthesiologists class I-II.

- Singleton full-term pregnancy.

- Voluntarily to receive caesarean section and postoperative controlled analgesia.

- Consented to participate in the study.

\section{Exclusion criteria}

- History of diagnosed mental disorders, psychiatric medication or psychotropic substance use at any time before.

- History of neurological diseases such as epilepsy.

- History of previously diagnosed depression.

- With suicidal ideation or history of suicide.

- History of drug, alcohol or opioid abuse.

- Monoamine oxidase inhibitor treatment at present or in the past 14 days.

- Participating in other clinical studies.

- With severe heart disease, brain disease, liver disease and kidney disease.

- With allergies to tramadol or opioids.

- With any contraindication for CSEA.

- Incapable of communication or cooperation.

Patients, who fail to follow the protocol of the study, have a lack of information regarding the main outcomes, or present serious complications during the process of caesarean section or hospitalisation will be excluded in the final analysis. The reasons for excluded cases will be explained in the case report form (CRF). In addition, the patients in the trial may choose to withdraw at any time during the study. Patients may refuse to continue the study and will be considered as having withdrawn, they may also be withdrawn at the discretion of the investigators for any safety reason. The reasons for the withdrawal will be explained in the CRF and the information will be maintained as accurately as possible. If the patient chooses to withdraw or refuses to consent to continuation, the data collected to the point of withdrawal of consent will be used as part of the intention-to-treat analysis.

\section{Randomisation}

Participants will be randomly allocated in a 1:1:1 ratio to receive PCIA using tramadol (group T), PCIA using hydromorphone (group H) or PCEA using ropivacaine (group R). Randomisation will be executed for each participant, by stratified block randomisation after the informed consent procedure is completed. The randomisation will be facilitated using SPSS V.17.0. As reported before, the strongest predictors of postpartum depression are history of depression during current pregnancy. ${ }^{64}$ Because screening for antenatal depression is not routinely performed in China and in many situations the pregnant women would not seek medical help even if they actually be accompanied by depressive symptom during pregnancy, at the time point of study recruitment it will be impossible to make an accurate judgement regarding patients' depressive state. However, to take the result of preoperative psychological test regarding depression as a stratified factor might be helpful to reduce the interference from antenatal depression status. Thus, in the exclusion criterion, we chose to exclude the women with known history of diagnosed mental disorders. And in the randomisation procedure whether or not with self-harm ideation or EPDS $\geq 10$ (a recommended cut-off score for screening depressive illness in Chinese general postnatal population ${ }^{45}$ ) in the preoperative psychological test was taken as stratified factor.

\section{Blinding}

In this study, because of the obvious difference between PCEA and PCIA treatments, total masking of the participant or the clinician during the surgery and hospitalisation is not feasible. However, the patients and investigator JP who perform postoperative follow-up will be blinded to the conditions of PCIA with tramadol or hydromorphone. Furthermore, investigator ZW who perform the long-term follow-up including psychiatric evaluations will be unaware of group allocations throughout the study. Additionally, when analysing the outcome data from the study, the study statistician will also be unaware of the groups until the end of the analysis.

\section{Intervention allocations}

Patient-controlled analgesic treatment will be applied using a controlled infusion pump. For patients in group $\mathrm{T}$, tramadol (Gran tai, Germany, $4 \mathrm{mg} / \mathrm{mL}$ ) mixed with $0.9 \%$ saline to a volume dose of $250 \mathrm{~mL}$ will be given through PCIA pump, in group $\mathrm{H}$, patients will receive hydromorphone (Renfu, China, $0.04 \mathrm{mg} / \mathrm{mL}$ ) mixed with $0.9 \%$ saline to a volume dose of $250 \mathrm{~mL}$, while in group $\mathrm{R}$, patients will receive ropivacaine (Astrazeneca, Sweden, $1.5 \mathrm{mg} / \mathrm{mL}$ ) mixed with $0.9 \%$ saline to a volume dose of $250 \mathrm{~mL}$. The PCIA pump $(250 \mathrm{~mL})$ will be programmed to use background infusion at $4.0 \mathrm{~mL} /$ hours, PCA dose of $1 \mathrm{~mL}$, lockout period of $15 \mathrm{~min}$. The PCEA pump $(250 \mathrm{~mL})$ will be programmed to use background infusion at $4.0 \mathrm{~mL} /$ hours, PCA dose of $4 \mathrm{~mL}$, lockout period of $15 \mathrm{~min}$. When inadequate treatment (rest pain visual analogue scale $[\mathrm{VAS}]>40$ ) is present during the postoperative analgesia, patients will be given a time-parameter setting adjustment for the PCIA pump. To prevent intraoperative or postoperative nausea and vomiting (PONV), metoclopramide $10 \mathrm{mg}$ and dexamethasone $10 \mathrm{mg}$ will be 
Table 1 Participant timeline in the study

\begin{tabular}{|c|c|c|c|c|c|}
\hline & Visit 1 & Visit 2 & Visit 3 & Visit 4 & Visit 5 \\
\hline Measurements & -1 week & Oweek & Hospitalisation & 4 weeks & 3 months \\
\hline Consent & $\sqrt{ }$ & & & & \\
\hline Screening & $\sqrt{ }$ & & & & \\
\hline Randomisation & $\sqrt{ }$ & & & & \\
\hline Demographics & $\sqrt{ }$ & & & & \\
\hline Gestational weeks & $\sqrt{ }$ & & & & \\
\hline Smoking status & $\sqrt{ }$ & & & & \\
\hline Alcohol status & $\sqrt{ }$ & & & & \\
\hline Preoperative complications & $\sqrt{ }$ & & & & \\
\hline Preoperative medications & $\sqrt{ }$ & & & & \\
\hline EPDS evaluation & $\sqrt{ }$ & & & $\sqrt{ }$ & $\sqrt{ }$ \\
\hline GAD-7 evaluation & $\sqrt{ }$ & & & $\sqrt{ }$ & $\sqrt{ }$ \\
\hline Blood collected & $\sqrt{ }$ & & $\sqrt{ }$ & & \\
\hline Surgery time & & $\sqrt{ }$ & & & \\
\hline Weight and height of the baby & & $\sqrt{ }$ & & & \\
\hline Apgar scores & & $\sqrt{ }$ & & & \\
\hline Blood loss & & $\sqrt{ }$ & & & \\
\hline Intraoperative complications & & $\sqrt{ }$ & & & \\
\hline Intraoperative medications & & $\sqrt{ }$ & & & \\
\hline Pain VAS evaluation & & & $\sqrt{ }$ & & \\
\hline Time to feel pain & & & $\sqrt{ }$ & & \\
\hline Early walking time & & & $\sqrt{ }$ & & \\
\hline Lactation onset time & & & $\sqrt{ }$ & & \\
\hline Postoperative complications & & & $\sqrt{ }$ & & \\
\hline Postoperative medications & & & $\sqrt{ }$ & & \\
\hline QoR-15 assessment & & & $\sqrt{ }$ & & \\
\hline Hospital stays & & & $\sqrt{ }$ & & \\
\hline Hospitalisation costs & & & $\sqrt{ }$ & & \\
\hline Psychiatric evaluations & & & & $\sqrt{ }$ & $\sqrt{ }$ \\
\hline Pain status & & & & $\sqrt{ }$ & $\sqrt{ }$ \\
\hline Postdischarge complications & & & & $\sqrt{ }$ & $\sqrt{ }$ \\
\hline Postdischarge medications & & & & $\sqrt{ }$ & $\sqrt{ }$ \\
\hline
\end{tabular}

EPDS, Edinburgh Postnatal Depression Scale; GAD-7, Generalised Anxiety Disorder 7-item; QoR-15, quality of recovery 15 items; VAS, Visual Analogue Scale.

given before the surgery for all patients. Although several previous studies have found that dexamethasone might be associated with depressive symptom, ${ }^{46}{ }^{47}$ at present its possible effect on postpartum depression remains unclear. Furthermore, in the study dexamethasone was given as single loading dose for prevention of PONV, and all patients in three groups will receive this same treatment. Thus, we speculate that possible mood effects of dexamethasone will not affect the research objective of the current study.

\section{Baseline data}

Both baseline data and study outcomes are displayed in a timed sequence in table 1 . Study participants will be interviewed by the investigator after they are recruited according to the inclusion and exclusion criteria. Preoperative data including demographics such as age, education level, household income, gestational weeks, smoking and alcohol status, contact information, known preoperative complications and medications, EPDS and GAD-7 will be collected. In addition intraoperative data including surgery time, weight and height of the baby, Apgar scores of the baby, blood loss, intraoperative complications and medications will be recorded.

The EPDS is a 10-item self-report depression questionnaire validated for use in both the perinatal and postnatal period $^{48} 49$ and its Chinese version has been validated 
as having satisfactory psychometric properties; a cut-off score of 10 is recommended for screening depressive illness in a general postnatal population. ${ }^{45}$ The GAD-7 has been used as a valuable screening tool for GAD in Chinese general hospital patients, and a score of $>9$ is considered indicative of the likely presence of GAD. ${ }^{5051}$ In the study, evaluation of EPDS and GAD-7 was trained and will be supervised by experienced psychiatric clinicians qualified with a master's degree.

\section{OUTCOMES}

\section{Primary outcomes}

In the study, incidence of postpartum depression at 4 weeks after the caesarean section is considered the primary outcome. All women will receive a web-based self-report EPDS form at 4 weeks after the surgery. Women who are screen positive (identified as EPDS $\geq 10$ or a positive response to question 10 , which is regarding self-harm) will be invited to receive psychiatric evaluations according to the SCID-5. When major depression is diagnosed, the patient will be analysed as a case positive for postpartum depression.

\section{Secondary outcomes}

In the study, secondary outcomes include incidence of postpartum depression at 3 months after the caesarean delivery, EPDS and GAD-7 scores at 4 weeks and 3 months after the caesarean delivery, postoperative Qor-15 items (Qor-15) score at 48 hours after the surgery and pain Visual Analogue Scale ( $0=$ no pain, $100=$ unbearable pain) for postoperative pain assessment at 6, 12, 24 and 48 hours after the surgery. The QoR-15 provides a valid, extensive and yet effective evaluation of postoperative Qor, ${ }^{52}$ and its Chinese version has been demonstrated to reveal satisfactory psychometric properties. ${ }^{53}$ In addition, a $3-5 \mathrm{~mL}$ peripheral venous blood sample for detection of norepinephrine and serotonin levels will be collected before the surgery and 48 hours after the analgesia treatment.

\section{Other outcomes}

The Ramsay sedation score will be recorded during the postoperative 48 hours follow-up. Early walking time (determined by the time point when patients could ambulate independently), lactation onset time will be recorded according to the patient's self-reported time points after the surgery. Self-reported sleep quality (rated as good or poor) on the day of surgery and 1 day after the surgery will also be evaluated. Postoperative adverse events including nausea and vomiting and pruritus will be noted. Additionally, the patients' length of hospital stay after the surgery and hospitalisation costs will be recorded. The patient's pain status and any complications of the mothers and their infants within 3 months after the surgery will be evaluated by structured telephone interview.

\section{Follow-up and data collection}

Study participants will complete a detailed survey before the caesarean section and also intraoperative and postoperative data will be recorded. All data will be collected as defined by the primary, secondary and other outcomes. Long-term data collection will be performed with follow-up phone calls at 4 weeks $( \pm 3$ days) and 3 months ( \pm 7 days) postdelivery. All data of the trial will be stored and only study investigators will have access to the final trial dataset.

\section{Sample size calculation}

The main outcome of the current trial is the incidence of postpartum depression at 4 weeks after the caesarean section. According to previous studies, the incidence of postpartum depression after the surgery in the Chinese population ranged from $7.3 \%$ to $26.3 \%,{ }^{54}$ and by combined calculation we speculate it is about $15 \%$ (table 2). Based on our previous pilot study results that postoperative HADS depression scores at 1 week after the surgery in tramadol group was lower than half that of hydromorphone group (GD, XB, GY, JP, ZW, PZ, ZZ, HL. The effect of tramadol on postoperative pain, anxiety and depression for patients undergoing secondary caesarean delivery: A randomised controlled trial), we hypothesised that PCIA with tramadol can reduce the $50 \%$ risk of postpartum depression compared with other analgesic strategies. Therefore, assuming an incidence of $7.5 \%$ in PCIA with tramadol and a $15 \%$ in both control interventions,

Table 2 A literature review of the incidence of postpartum depression in women undergoing caesarean section in the Chinese population

\begin{tabular}{|c|c|c|c|c|c|}
\hline & Diagnostic criteria & Observed time point & Positive cases & Included cases & Incidence, \% \\
\hline Wang et $\left.a\right|^{54}$ & $E P D S \geq 13$ & PO 6 weeks & 52 & 710 & 7.3 \\
\hline Liu et $a l^{56}$ & $E P D S \geq 13$ & PO 6 weeks & 44 & 198 & 22.2 \\
\hline Xie et $a l^{57}$ & $E P D S \geq 13$ & PO 2 weeks & 90 & 415 & 21.7 \\
\hline $\mathrm{He}$ and $\mathrm{GP}^{58}$ & $E P D S \geq 13$ & PO 6 weeks & 84 & 320 & 26.3 \\
\hline Zhang et a ${ }^{59}$ & $S D S \geq 63$ & PO 1 week & 22 & 300 & 7.3 \\
\hline Tam et $a /^{60}$ & $\mathrm{GHQ} \geq 5$ & PO 6 weeks & 35 & 255 & 13.7 \\
\hline Sum & NA & NA & 327 & 2198 & 14.9 \\
\hline
\end{tabular}

EPDS, Edinburgh Postnatal Depression Scale; GHQ, General Health Questionnaire; NA, not applicable; PO, postoperative ; SDS, Self-Rating Depression Scale. 
based on a significance level of 0.05 , power of 0.9 and considering about $10 \%$ loss of follow-up, the required minimum sample size is 410 for each group.

\section{Statistical analysis}

To ensure high quality study conduct, data entry will be carried out using Epidata V.3.1; then data cleaning, coding and analysis will be performed using SPSS V.17.0 statistical software. Standard descriptive statistics, such as the mean (SD), number (frequency) and median (IQR), will be used to summarise the variables. Two-tailed $\mathrm{p}<0.05$ is considered statistically significant. Outcome data will be analysed in the intention-to-treat population, and we will also perform per-protocol analysis for the primary endpoint. The primary outcome analysis of the study is to compare the incidence of postpartum depression at 4 weeks after the surgery in group $\mathrm{T}$ with group $\mathrm{H}$ and group R, respectively. A subgroup comparison according to whether or not the preoperative EPDS $\geq 10$ will be performed. Categorical data including the incidence of postpartum depression will be analysed using $\chi^{2}$ test, continuity correction the $\chi^{2}$ test or Fisher's exact test. Comparison between group $\mathrm{T}$ and group $\mathrm{H}$, and comparison between group $\mathrm{T}$ and group $\mathrm{R}$ will be performed according to Bonferroni correction $\mathrm{p}$ value of $0.05 / 2$. The rate difference with $95 \%$ CI between different groups will be calculated. Normally distributed continuous data between different groups will be compared using analysis of variance (ANOVA) analysis with post hoc least significant difference test. Data of abnormal distribution will be compared using the Kruskal-Wallis test. Two-way repeated ANOVA analysis will be applied to analyse the repeated measures at different time points among different groups.

\section{Patient and public involvement}

No patient or public was involved in study design or conduct of the current study. All participants will be informed about the study results in an international peer-reviewed scientific journal on the completion of the study.

\section{Ethics and dissemination}

.In the study, all participants will be recruited in the antenatal period prior to caesarean delivery and informed consent (online supplementary file 1) will be obtained from all patients prior to the study enrolment.

According to the study inclusion and exclusion criteria, study investigator will daily review the parturients who are admitted to the obstetric ward and identify the potentially eligible women patients. Then the investigator will inform the potential women patients about the background and aims of the current trial. And informed consent will be given for individuals who are interested in enrolling in the study. Study investigator will ensure that parturients understand all items outlined in the consent form prior to signing written consent and voluntary participation. In particular, all women patients will be informed that one of three potential analgesic strategy will be randomly applied. And patients will receive information to minimise their anxiety related to the different analgesic strategy. Adverse events will be tracked and treated promptly, and reporting will occur following institutional guidelines.

\section{Dissemination}

The current trial data will be analysed and disseminated at scientific meetings, and in international peer-reviewed scientific journals. Safety and adverse event data will be reported to the supervisory authority where appropriate.

\section{Monitoring}

The progress of the study and overall conduct of the clinical trial will be monitored by data monitoring committee (DMC). The DMC consists of an independent chair, clinical representative, patient representative and statistician. They will meet at regular intervals throughout the study period as determined by the chair, but as a minimum at least once every 12 months.

\section{DISCUSSION}

The study aimed to explore the potential effect of intravenous tramadol on postpartum depression for patients who are receiving caesarean section. Because antenatal depression is the strongest identified predictors of postpartum depression, ${ }^{64}$ it will be necessary to take this into account as a stratified factor when randomisation is performed. However, screening for antenatal and postpartum depression is not routinely performed in China. Therefore, in this trial EPDS will be preoperatively evaluated when the patients are recruited, and whether or not EPDS $\geq 10$ in the preoperative psychological test will be taken as a stratified factor. By doing this, we can exclude any interference from antenatal depression status and furthermore this will make it possible to perform a subgroup analysis to test the possible effect of tramadol in caesarean patients with different antenatal psychological status.

In the current study, all preoperative and intraoperative procedures are performed according to the judgements of anaesthesiologists and obstetricians based on conventional clinical guidelines. During the postoperative period, PCIA with tramadol, PCIA with hydromorphone or PCEA with ropivacaine will be randomly given for the postoperative analgesia. Regarding the control group allocations for PCIA tramadol, the current trial includes two types of analgesic methods (PCIA with hydromorphone and PCEA with ropivacaine), all of which are commonly used analgesic strategies after a caesarean section. In this trial, PCIA with hydromorphone will be taken as a control that issuing the same mode of administration but with different analgesic. While PCEA with ropivacaine will be taken as another control that issuing a different mode of administration. Because both of these two groups are considered as control methods, the main comparisons will be the difference between PCIA with tramadol, and PCIA with hydromorphone and PCEA with ropivacaine. 
As reported in the International Statistical Classification of Diseases, 10th revision, postpartum depression is defined as depression onset within 6 weeks post partum. ${ }^{2}$ In contrast, in the DSM-5, the definition includes onset of symptoms during pregnancy and for up to 4 weeks post partum. ${ }^{2}$ Therefore, at present the diagnostic definition of postpartum depression remains a topic of debate, various temporal definitions have been proposed. ${ }^{55}$ In the current trial, we explored and focused on the possible effect of analgesic treatment on postpartum depression during the postoperative 48 hours. However, the possible effect duration of postoperative tramadol treatment is unknown, and we speculated that positive effect may be more likely to present at 4 week after caesarean section than that at 6 week. Thus, we chose the postpartum time frame to perform the psychological assessment, and the incidence of postpartum depression at 4 weeks after the caesarean section will be taken as the primary outcome.

Acknowledgements The authors thank all patients and patient advisers for their contribution to the trail.

Contributors GD and $\mathrm{HL}$ conceived of the study question, hypothesis, carried out the evaluation of current literature, conceptualised the methodology and analysis, drafted and reviewed the manuscript. ZW and PZ conceptualised the methodology and assisted with manuscript preparation. JP, ZC and QZ assisted with the methodology and reviewed the manuscript. RX assisted in analysis and reviewed the manuscript.

Funding The study was supported by Clinical Research Projects of Xinqiao Hospital, Third Military Medical University (No. 2016YLC10 and 2015YLC09).

Competing interests None declared.

Patient consent Obtained.

Ethics approval The current study protocol was approved by the Institutional Ethics Committee of Xinqiao Hospital (ID: 2017-026), Third Military Medical University, Chongqing, China.

Provenance and peer review Not commissioned; externally peer reviewed.

Open access This is an open access article distributed in accordance with the Creative Commons Attribution Non Commercial (CC BY-NC 4.0) license, which permits others to distribute, remix, adapt, build upon this work non-commercially, and license their derivative works on different terms, provided the original work is properly cited, appropriate credit is given, any changes made indicated, and the use is non-commercial. See: http://creativecommons.org/licenses/by-nc/4.0/.

\section{REFERENCES}

1. Stewart DE, Vigod S. Postpartum depression. N Engl J Med 2016;375:2177-86.

2. Howard LM, Molyneaux E, Dennis CL, et al. Non-psychotic mental disorders in the perinatal period. Lancet 2014;384:1775-88.

3. Munk-Olsen T, Laursen TM, Pedersen CB, et al. New parents and mental disorders: a population-based register study. JAMA 2006;296:2582-9.

4. Siu BW, Leung SS, Ip P, et al. Antenatal risk factors for postnatal depression: a prospective study of Chinese women at maternal and child health centres. BMC Psychiatry 2012;12:22.

5. Wang SY, Jiang XY, Jan WC, et al. A comparative study of postnatal depression and its predictors in Taiwan and mainland China. Am J Obstet Gynecol 2003;189:1407-12.

6. Wisner KL, Sit DK, McShea MC, et al. Onset timing, thoughts of self-harm, and diagnoses in postpartum women with screen-positive depression findings. JAMA Psychiatry 2013;70:490-8.

7. Stein A, Pearson RM, Goodman SH, et al. Effects of perinatal mental disorders on the fetus and child. Lancet 2014;384:1800-19.

8. Goodman JH. Postpartum depression beyond the early postpartum period. J Obstet Gynecol Neonatal Nurs 2004;33:410-20.

9. Howard LM, Piot P, Stein A. No health without perinatal mental health. Lancet 2014;384:1723-4.
10. Lumbiganon P, Laopaiboon M, Gülmezoglu AM, et al. Method of delivery and pregnancy outcomes in Asia: the WHO global survey on maternal and perinatal health 2007-08. Lancet 2010;375:490-9.

11. Li HT, Luo S, Trasande L, et al. Geographic Variations and Temporal Trends in Cesarean Delivery Rates in China, 2008-2014. JAMA 2017;317:69-76.

12. Hamilton BE, Martin JA, Osterman MJ, et al. Births: Preliminary Data for 2014. Natl Vital Stat Rep 2015;64:1-19.

13. Meltzer-Brody S, Maegbaek ML, Medland SE, et al. Obstetrical, pregnancy and socio-economic predictors for new-onset severe postpartum psychiatric disorders in primiparous women. Psychol Med 2017;47:1427-41.

14. Sword W, Landy CK, Thabane L, et al. Is mode of delivery associated with postpartum depression at 6 weeks: a prospective cohort study. BJOG 2011;118:966-77.

15. Xie R-hua, Lei J, Wang S, et al. Cesarean Section and Postpartum Depression in a Cohort of Chinese Women with a High Cesarean Delivery Rate. J Womens Health 2011;20:1881-6.

16. Lobel M, DeLuca RS. Psychosocial sequelae of cesarean delivery: review and analysis of their causes and implications. Soc Sci Med 2007;64:2272-84.

17. Wiklund I, Edman G, Andolf E. Cesarean section on maternal request: reasons for the request, self-estimated health, expectations, experience of birth and signs of depression among first-time mothers. Acta Obstet Gynecol Scand 2007;86:451-6.

18. McDonnell NJ, Keating ML, Muchatuta NA, et al. Analgesia after caesarean delivery. Anaesth Intensive Care 2009;37:539-51.

19. Bloch M, Schmidt PJ, Danaceau M, et al. Effects of gonadal steroids in women with a history of postpartum depression. Am J Psychiatry 2000;157:924-30.

20. Mehta D, Newport DJ, Frishman G, et al. Early predictive biomarkers for postpartum depression point to a role for estrogen receptor signaling. Psychol Med 2014;44:2309-22.

21. Guintivano J, Arad M, Gould TD, et al. Antenatal prediction of postpartum depression with blood DNA methylation biomarkers. Mol Psychiatry 2014;19:560-7.

22. Couto TC, Brancaglion MY, Alvim-Soares A, et al. Postpartum depression: A systematic review of the genetics involved. World $\mathrm{J}$ Psychiatry 2015;5:103-11.

23. Dennis CL, Hodnett E. Psychosocial and psychological interventions for treating postpartum depression. Cochrane Database Syst Rev 2007:CD006116. CD006116.

24. Gentile S. Use of contemporary antidepressants during breastfeeding: a proposal for a specific safety index. Drug Saf 2007;30:107-21.

25. Molyneaux E, Howard LM, McGeown HR, et al. Antidepressant treatment for postnatal depression. Cochrane Database Syst Rev 2014:CD002018. CD002018.

26. Gerbershagen HJ, Aduckathil S, van Wijck AJ, et al. Pain intensity on the first day after surgery: a prospective cohort study comparing 179 surgical procedures. Anesthesiology 2013;118:934-44.

27. Kagwa S, Hoeft MA, Firth PG, et al. Ultrasound guided transversus abdominis plane versus sham blocks after caesarean section in an Ugandan village hospital: a prospective, randomised, doubleblinded, single-centre study. Lancet 2015;385:S36.

28. Eisenach JC, Pan PH, Smiley R, et al. Severity of acute pain after childbirth, but not type of delivery, predicts persistent pain and postpartum depression. Pain 2008;140:87-94.

29. Costa JR, Coleman R. Post-operative pain management using patient-controlled analgesia. Clin Podiatr Med Surg 2008;25:465-75.

30. Alhashemi JA, Alotaibi QA, Mashaat MS, et al. Intravenous acetaminophen vs oral ibuprofen in combination with morphine PCIA after Cesarean delivery. Can J Anaesth 2006;53:1200-6.

31. Lehmann KA, Paral F, Sabatowski R. [Postoperative pain therapy with hydromorphone and metamizole. A prospective randomized study in intravenous patient-controlled analgesia (PCA)]. Anaesthesist 2001;50:750-6.

32. Scott LJ, Perry CM. Tramadol: a review of its use in perioperative pain. Drugs 2000;60:139-76.

33. Verstraete S, Van de Velde M. Post-cesarean section analgesia. Acta Anaesthesiol Belg 2012;63:147-67.

34. Demirel I, Ozer AB, Atilgan R, et al. Comparison of patient-controlled analgesia versus continuous infusion of tramadol in post-cesarean section pain management. J Obstet Gynaecol Res 2014;40:392-8.

35. Kanazi GE, Aouad MT, Abdallah FW, et al. The analgesic efficacy of subarachnoid morphine in comparison with ultrasound-guided transversus abdominis plane block after cesarean delivery: a randomized controlled trial. Anesth Analg 2010;111:475-81.

36. Mitra S, Khandelwal P, Sehgal A. Diclofenac-tramadol vs. diclofenacacetaminophen combinations for pain relief after caesarean section. Acta Anaesthesiol Scand 2012;56:706-11. 
37. Chi X, Li M, Mei W, et al. Comparison of patient-controlled intravenous analgesia with sufentanil versus tramadol in postcesarean section pain management and lactation after general anesthesia - a prospective, randomized, double-blind, controlled study. J Pain Res 2017;10:1521-7.

38. Aishwarya S, Rajendiren S, Kattimani S, et al. Homocysteine and serotonin: association with postpartum depression. Asian J Psychiatr 2013;6:473-7.

39. Treadway CR, Kane FJ, Jarrahi-Zadeh A, et al. A psychoendocrine study of pregnancy and puerperium. Am J Psychiatry 1969;125:1380-6.

40. Orsolini L, Bellantuono C. Serotonin reuptake inhibitors and breastfeeding: a systematic review. Hum Psychopharmacol 2015;30:4-20.

41. Nccf UK. Antenatal and postnatal mental health: clinical management and service guidance: updated edition. Leicester, UK: British Psychological Society, 2014.

42. Schulz KF, Altman DG, Moher D. CONSORT Group. CONSORT 2010 statement: updated guidelines for reporting parallel group randomised trials. PLoS Med 2010;7:e1000251.

43. UK NCCF. Antenatal and Postnatal Mental Health: Clinical Management and Service Guidance. Leicester UK: British Psychological Society, 2014.

44. Postpartum Depression: Action Towards Causes and Treatment (PACT) Consortium. Heterogeneity of postpartum depression: a latent class analysis. Lancet Psychiatry 2015;2:59-67.

45. Lee DT, Yip SK, Chiu HF, et al. Detecting postnatal depression in Chinese women. Validation of the Chinese version of the Edinburgh Postnatal Depression Scale. Br J Psychiatry 1998;172:433-7.

46. Dunlop BW, Betancourt Y, Binder EB, et al. Tolerability of the dexamethasone-corticotropin releasing hormone test in major depressive disorder. J Psychiatr Res 2011;45:24-8.

47. Tsai HC, Lin $\mathrm{SH}$, Lee IH, et al. Probing the association between dexamethasone-induced cortisol suppression and serotonin transporter availability among drug-free patients with major depressive disorder-a small-sample SPECT study with [123|]ADAM. Psychoneuroendocrinology 2013;38:2805-9.
48. Patton GC, Romaniuk H, Spry E, et al. Prediction of perinatal depression from adolescence and before conception (VIHCS): 20year prospective cohort study. Lancet 2015;386:875-83.

49. Rojas G, Fritsch R, Solis J, et al. Treatment of postnatal depression in low-income mothers in primary-care clinics in Santiago, Chile: a randomised controlled trial. Lancet 2007;370:1629-37.

50. Tong X, An D, McGonigal A, et al. Validation of the Generalized Anxiety Disorder-7 (GAD-7) among Chinese people with epilepsy. Epilepsy Res 2016;120:31-6.

51. He X. Reliability and validity of a generalized anxiety disorder scale in general hospital outpatients. Shanghai Archives of Psychiatry 2010;4:200-3.

52. Stark PA, Myles PS, Burke JA. Development and psychometric evaluation of a postoperative quality of recovery score: the QoR-15. Anesthesiology 2013;118:1332-40.

53. Bu XS, Zhang J, Zuo YX. Validation of the Chinese Version of the Quality of Recovery-15 Score and its comparison with the postoperative quality recovery scale. Patient 2016;9:251-9.

54. Wang SY, Duan KM, Tan XF, et al. Genetic variants of the kynurenine-3-monooxygenase and postpartum depressive symptoms after cesarean section in Chinese women. J Affect Disord 2017;215:94-101.

55. Wisner KL, Moses-Kolko EL, Sit DK. Postpartum depression: a disorder in search of a definition. Arch Womens Ment Health 2010;13:37-40.

56. Liu YQ, Maloni JA, Petrini MA. Effect of postpartum practices of doing the month on Chinese women's physical and psychological health. Biol Res Nurs 2014;16:55-63.

57. Xie RH, Lei J, Wang S, et al. Cesarean section and postpartum depression in a cohort of Chinese women with a high cesarean delivery rate. J Womens Health 2011;20:1881-6.

58. He Y, GP H. A cross-sectional study of postpartum depression in Chinese puerpera. Chin J Mod Nurs 2011;13:1539-41.

59. Zhang Y, Gq Z. JL H. A Study On the Postpartum Depression of The Patients Of Caesarean Birth anditsrelated factors. China Journal of Health Psychology 2008;8:946-7.

60. Tam WH, Lee DT, Chiu HF, et al. A randomised controlled trial of educational counselling on the management of women who have suffered suboptimal outcomes in pregnancy. BJOG 2003;110:853-9. 